\title{
Implementation of a New Clinic-Based, Pharmacist-Managed PCSK9 Inhibitor Consultation Service
}

\author{
Adenike Atanda, PharmD, CTTS, BCACP; Nancy L. Shapiro, PharmD, FCCP, BCACP; \\ JoAnn Stubbings, BSPharm, MHCA; and Vicki Groo, PharmD
}

\begin{abstract}
BACKGROUND: The proprotein convertase subtilisin/kexin type 9 (PCSK9) inhibitors alirocumab and evolocumab were approved by the FDA in 2015. In anticipation of provider interest and a potential increase in referrals to the on-site specialty pharmacy, we created a pharmacist-managed consultation service.

PROGRAM DESCRIPTION: The development of a clinic-based pharmacistmanaged consultation service for the management of the PCSK9 inhibitor agents alirocumab and evolocumab is described. Key implementation steps included (a) creation of a pharmacy team and collaboration with cardiology; (b) completion of a needs assessment; (c) service creation; (d) collaboration with the on-site specialty pharmacy; (e) development of an electronic consult order and consult pool; (f) personnel training; and (g) service approval and marketing. The service development occurred over 9 months (July 2015-April 2016) and was implemented hospital-wide in May 2016.

OBSERVATIONS: The University of Illinois Hospital and Health Sciences System PCSK9 inhibitor consultation service successfully integrated the benefits of a clinical review process, information technology capabilities of an electronic medical record system, and collaboration with the on-site specialty pharmacy to provide a comprehensive service that aimed to facilitate appropriate medication management from prescribing to patient administration and provide monitoring for this class of specialty medications.
\end{abstract}

IMPLICATIONS/RECOMMENDATIONS: The PCSK9 pharmacist-managed consultation service provides a method for complex therapies to be managed comprehensively through the collaboration of ambulatory care clinics and outpatient specialty pharmacies.

J Manag Care Spec Pharm. 2017;23(9):918-25

Copyright $\odot 2017$, Academy of Managed Care Pharmacy. All rights reserved.

\section{What is already known about this subject}

The proprotein convertase subtilisin/kexin type 9 (PCSK9) inhibitors are new injectable drugs that are used to lower cholesterol. Health system-based specialty pharmacy practice models have been documented in the literature.

PCSK9 inhibitors require prior authorization from insurance companies.

\section{What this study adds}

A new service model of integrating clinical and specialty pharmacy to proactively manage medications is described.

An electronic consult order was built into the electronic medical record and attached to an electronic prescription for a PCSK9 inhibitor to initiate the service. he proprotein convertase subtilisin/kexin type 9 (PCSK9) inhibitors alirocumab and evolocumab are monoclonal antibodies approved for the treatment of patients with familial hypercholesterolemia (FH) or clinical atherosclerotic cardiovascular disease (ASCVD), who require additional low-density lipoprotein (LDL) cholesterol lowering as an adjunct to diet and maximally tolerated statin therapy. ${ }^{1,2}$ These agents lower LDL levels by preventing the PCSK9mediated degradation of LDL receptors in hepatocytes. In clinical trials, alirocumab and evolocumab lower serum LDL levels by approximately 50\% when used as monotherapy or adjunct to statin therapy. ${ }^{3-8}$ They are administered by subcutaneous injection every 2-4 weeks. The most common adverse events reported are local injection site reactions, upper respiratory tract infections, and neurocognitive events. ${ }^{3-8}$

Currently, statin therapy is the mainstay of treatment for hypercholesterolemia because of the availability of long-term data supporting its efficacy and safety profile. ${ }^{9-12}$ However, statin therapy can be associated with myopathy, rhabdomyolysis, and hepatic impairment, which limits medication titration or its use. Ezetimibe and bile acid sequestrants, such as colesevelam, cholestyramine, and colestipol, are used as monotherapy or combination therapy with statins in the management of ASCVD as highlighted in the American College of Cardiology (ACC) document "2016 ACC Expert Consensus Decision Pathway on the Role of Non-Statin Therapies for LDLCholesterol Lowering in the Management of Atherosclerotic Cardiovascular Disease Risk."13-15 Although PCSK9 inhibitors offer the benefit of additional LDL lowering, their use is limited by several factors, including cost, lack of long-term cardiovascular or safety data, and special claims processing requirements.

The University of Illinois Hospital and Health Sciences System (UIH) is an academic health system that includes a 400-bed safety net hospital, 23 outpatient clinics, and an integrated on-site specialty and mail order pharmacy. The on-site specialty and mail order pharmacy manages approximately 700 patients from the oncology, hepatology, neurology, rheumatology, gastrointestinal disease, sickle cell disease, transplant, women's health, pulmonary arterial hypertension, and cardiology clinics. Services include insurance benefit investigation and coordination, treatment initiation, treatment education and injection teaching, adverse event monitoring, 
coordination of care with the UIH specialty outpatient clinics, refill management, and medication delivery services.

The UIH outpatient specialty pharmacy integrates the 4 main components of specialty pharmacy care (referral management, medication access, fulfillment, and care plans) into the health system via a closed loop workflow. The electronic medical record (EMR) serves as the primary mode of communication between the specialty pharmacy and the clinics. The pharmacy received accreditation from the Utilization Review Accreditation Commission in September 2015 and the Accreditation Commission for Health Care in December 2016.

PCSK9 inhibitors are available through public or private insurance at approximately $\$ 15,000$ per patient per year. Prior authorization (PA) approval is required, and the drug may be dispensed through a specialty pharmacy. Individual health care providers in the outpatient clinics are responsible for submitting PA requests for the PCSK9 inhibitors directly to insurance companies, often using the patient enrollment forms from the product manufacturers. Patient enrollment forms are designed to facilitate insurance support, financial assistance, and injection training for a brand-name drug. However, this process usually requires multiple steps that can lead to claim denials because of incomplete clinical review and documentation, resulting in loss of valuable time that should be spent on direct patient care services.

In anticipation of provider interest and the potential for increased specialty pharmacy prescription volume, UIH created a PCSK9 pharmacist-managed consultation service. The purpose of the service was to ensure appropriate prescribing, expedite access by streamlining the PA process, provide adequate patient education, and enhance collaboration between the UIH outpatient clinics and the on-site specialty pharmacy. The purpose of this article was to describe the process of service development and report our initial experience.

\section{Program Description}

During July 2015, we created a PCSK9 pharmacy team consisting of 5 clinical pharmacists, a postgraduate year 2 (PGY2) ambulatory care pharmacy resident, and 3 specialty pharmacists from the UIH outpatient specialty pharmacy. The clinical pharmacists practice in UIH outpatient cardiology, family medicine, and internal medicine clinics, since it was thought that they would have the highest potential patient population for the PCSK9 inhibitor consultation service. Two cardiologists from the UIH department of cardiology functioned as physician advocates to ensure that the consultation service adequately captured the needs of the providers.

After the creation of the pharmacy team, we conducted a needs assessment in the form of a chart review. We identified 108 patients with an LDL cholesterol level greater than $190 \mathrm{mg} / \mathrm{dL}$, of which 29\% were taking a high-intensity statin and 22\% were taking a medium-intensity statin. Seventy-five percent of the patients were managed by their primary care providers; $14 \%$ were managed by their endocrinologists; and $11 \%$ were managed by their cardiologists. These data confirmed a potential for the prescribing of PCSK9 inhibitors.

Next, we developed the structure of the pharmacist-managed consultation service. The service model includes 3 key elements: (1) a clinical review to screen patients for eligibility and offer recommendations for alternative therapies for ineligible patients; (2) collaboration with the on-site specialty pharmacy to facilitate claims processing, treatment initiation, and medication delivery for the PCSK9 inhibitor agents; and (3) creation of an electronic consult order built into the EMR and attached to an electronic prescription for alirocumab or evolocumab. Other key aspects included training the clinical pharmacists and pharmacy residents to ensure a standardized implementation process and formalizing a communication procedure for presenting the new service to providers.

\section{Clinical Review}

Clinical pharmacists, the pharmacy resident from the PCSK9 pharmacy team, and a cardiologist collaborated to create the clinical review process. Key concerns outlined by the clinical review subgroup included appropriateness of PCSK9 inhibitor therapy, titration of statin therapy to maximum tolerated doses, adequate documentation of statin intolerance or adverse events, and creation of a clinical note template to document recommendations and interventions made by the team. To assist with appropriate prescribing of the PCSK9 inhibitor agents, providers and consulting pharmacists are encouraged to comply with the position of the ACC decision pathway on nonstatin therapies. ${ }^{14}$

The clinical review process involves screening a patient's chart for the following pertinent information: age, indication (i.e., FH, ASCVD history), serum lipid levels, current statin therapy, statin history, documentation of statin contraindication (intolerance or adverse event), and latex allergy (because of evolocumab's needle cover). This information is documented in the EMR using the clinical note template, along with recommendations, and forwarded to the patient's physician. A template note ensures appropriate and uniform documentation of recommendations and interventions. The information in the clinical note is used in the PA submission process. In addition, the clinical pharmacist contacts the patient regarding the drug therapy, insurance process, and to answer any questions or concerns.

Pharmacy residents are assigned to complete the clinical review process under direct supervision of the clinical pharmacists on the PCSK9 pharmacy team. The clinical pharmacists review the appropriateness of therapy recommendations and cosign the progress note. Pharmacy residents are given more autonomy as their residency year progresses to allow independent management of the consultation service. The pharmacy residents are supervised for 3 months and then allowed to 


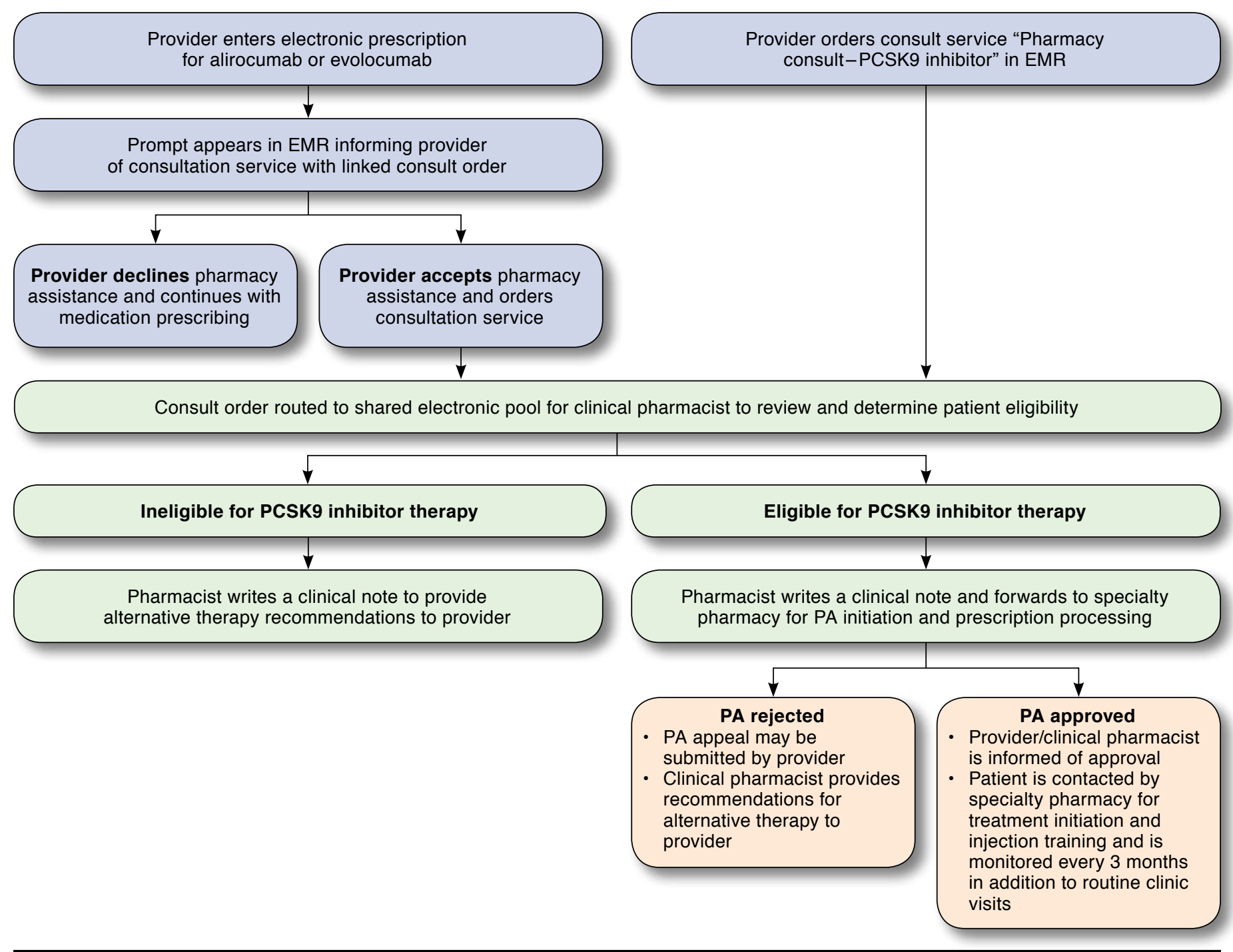

EMR=electronic medical record; $P A=$ prior authorization.

operate autonomously if they are able to provide appropriate treatment recommendations and implement the consultation service with minimal assistance.

\section{Collaboration with the Specialty Pharmacy}

In the United States, specialty pharmacies manage the dispensing of PCSK9 inhibitor agents because of their high cost, storage requirements, and need for self-injection training. Collaboration with the on-site UIH specialty pharmacy allows patients to benefit from the proximity of the pharmacy and close communication between the pharmacy and the $\mathrm{UIH}$ outpatient clinics. Pharmacists from the PCSK9 consultation service refer patients to the UIH on-site specialty pharmacy via the clinical review note created in the EMR. The specialty pharmacy uses the information provided for benefit investigation and PA submission. After insurance approval, the specialty pharmacists contact the patient to provide education, injection training, apply financial assistance, and arrange for medication pickup or delivery. Follow-up services include medication monitoring and refill management. If the UIC specialty pharmacy cannot fill the prescription because of network restrictions, or if the patient chooses to receive the prescription from another pharmacy, the prescription is transferred to an outside pharmacy of the patient's choice. The clinical pharmacist assists the 
outside pharmacy with patient information needed for PA processing, patient education, injection training, and monitoring, as needed.

\section{Development of Electronic Consult Order Form and Consult Pool}

The PCSK9 pharmacy team collaborated with the UIH Information Technology (IT) department to create an electronic consult form, link the form to electronic prescriptions for alirocumab and evolocumab, and create an electronic pharmacy pool for the management of referred patients. All tasks and forms were approved by the UIH Documentation Committee and the UIH Rules and Orders Hospital Committee.

The electronic consult form captures the following information: patient demographic (name and medical record number), name of requesting provider, desired PCSK9 inhibitor agent, and if the patient is currently on statin therapy. The consult form is available as a stand-alone order and is linked to the PCSK9 inhibitors through a prompt triggered by an electronic prescription for either alirocumab or evolocumab. The prompt informs the provider of the consultation service and has the consult order preselected to allow a 1-step process. The prompt is not created as a hard stop but allows providers to decline assistance from the PCSK9 consultation service using a 3-step process. The provider has to unselect the consult order, select the option to decline pharmacy assistance, and select "OK" to proceed without pharmacy assistance (Figure 1).

Finally, the consults are stored in a pool in the EMR message center. The clinical pharmacists and pharmacy residents on the team have access to the pool. This consult pool was subsequently converted into a general "pharmacy consult pool" to enable the creation and facilitation of consultation services for other specialty medications. The consults are given unique tags (e.g., "Pharmacy consult-PCSK9 inhibitor") to allow easy identification of consult orders in the pool. Clinical pharmacists and pharmacy residents only process consult orders for their respective services. All patient information and interventions are documented within the EMR to ensure compliance with the Health Insurance Portability and Accountability Act (HIPAA) privacy rules.

\section{Service Personnel Training}

A comprehensive implementation guide was created to facilitate appropriate training of service personnel. The guide includes contact information, instructions for service implementation, background information about the PCSK9 inhibitors, the ACC decision pathway for nonstatin therapies, ${ }^{14}$ and the clinical note template. The clinical note documents the requesting provider, history of present illness, past medical history, allergies, medication list, PCSK9 inhibitor indication, lipid levels, statin history, statin intolerance if applicable, assessment, and recommendations. The PCSK9 pharmacy team also met with pharmaceutical representatives of alirocumab and evolocumab to receive injection training materials and patient education materials.

The consultation service is primarily managed by pharmacy residents under the supervision of the PCSK9 clinical pharmacist team. Pharmacy residents receive training from the pharmacists and have the service implementation guide as a resource. UIH pharmacy residents are expected to provide daily coverage of the service on weekdays from 9 am to $5 \mathrm{pm}$ with a desired turnaround time of 48 hours for the initial consult note. Pharmacy residents (e.g., PGY2 ambulatory care residents, PGY2 cardiology residents, and PGY1 specialty pharmacy residents) provide coverage on a monthly basis. The residents review patient charts in the EMR and document recommendations in the standardized clinic note template, which will be forwarded to the clinical pharmacist for review and to the requesting provider for cosignature. The clinical pharmacists provide oversight of the pharmacy residents by reviewing clinical notes and interventions before they are sent to the providers. The clinical pharmacists and residents are also responsible for completing PA claims for patients whose insurance provider does not allow the prescription to be filled at the UIH specialty pharmacy.

\section{Service Approval and Marketing}

Details of the consultation service were presented at key department and section meetings (e.g., ambulatory pharmacy and cardiology meetings). The service received approval from the following committees and departments: UIH Department of Pharmacy Administration and Clinical Service Managers, UIH Section of Cardiology, and the UIH Pharmacy and Therapeutics Committee.

The director of pharmacy sent an announcement email to all clinical staff in the UIH health system in May 2016. The email included a PCSK9 inhibitor treatment algorithm (adapted from the ACC decision pathway on nonstatin therapies ${ }^{14}$ ) and education about the service. The education outlined indications approved by the U.S. Food and Drug Administration (FDA), purpose of the service, how to place a consult in the EMR, and what to expect from the clinical pharmacists and the specialty pharmacy. The consult order form linked to the PCSK9 inhibitor prescription also contains details of the service.

\section{Observations}

\section{Consultation Experience}

The PCSK9 inhibitor consultation service was launched to all UIH providers in May 2016 after a successful pilot in the cardiology clinic. Based on a prescribing report, no prescriptions bypassed the consultation service. However, if a prescription was initiated using the pharmaceutical company patient enrollment forms and not entered into the EMR, it was not captured in this report. 


\begin{tabular}{|c|c|c|c|c|c|c|}
\hline Patients & Service & Indication & Lipid Therapy & LDL (mg/dL) & $\begin{array}{l}\text { Pharmacist } \\
\text { Recommendation }\end{array}$ & Outcome \\
\hline 1 & Cardiology & $\begin{array}{l}\mathrm{FH} / \text { statin } \\
\text { intolerance }\end{array}$ & none & 233 & $\begin{array}{l}\text { Start ezetimibe or } \\
\text { PCSK9 inhibitor }\end{array}$ & $\begin{array}{l}\mathrm{LDL}=151 \mathrm{mg} / \mathrm{dL} \text { on alirocumaba } \\
\text { Self-discontinued due to side effects }\end{array}$ \\
\hline 2 & Cardiology & $\begin{array}{l}\text { ASCVD; } \\
\text { multiple PCI }\end{array}$ & $\begin{array}{l}\text { Atorvastatin } \\
80 \mathrm{mg} \text { once daily }\end{array}$ & 124 & $\begin{array}{l}\text { Start PCSK9 } \\
\text { inhibitor }\end{array}$ & $\mathrm{LDL}=30 \mathrm{mg} / \mathrm{dL}$ on alirocumaba \\
\hline 3 & Cardiology & $\begin{array}{l}\text { ASCVD; } \\
\text { multiple PCI }\end{array}$ & $\begin{array}{l}\text { Rosuvastatin } \\
20 \text { mg once daily }\end{array}$ & 86 & $\begin{array}{l}\text { Increase } \\
\text { rosuvastatin } \\
\text { or start PCSK9 } \\
\text { inhibitor }\end{array}$ & $\mathrm{LDL}=40 \mathrm{mg} / \mathrm{dL}$ on alirocumaba \\
\hline 4 & Cardiology & $\begin{array}{l}\text { Family ASCVD } \\
\text { history, elevated } \\
\text { coronary artery } \\
\text { calcium score }\end{array}$ & $\begin{array}{l}\text { Rosuvastatin } \\
40 \text { mg once daily }\end{array}$ & 86 & Not indicated & $\begin{array}{l}\text { LDL } 86 \mathrm{mg} / \mathrm{dL} \text { on rosuvastatin } 40 \mathrm{mg} \text { daily } \\
\text { No additional recommendations made }\end{array}$ \\
\hline 5 & Cardiology & $\begin{array}{l}\text { ASCVD; } \\
\text { multiple PCI }\end{array}$ & $\begin{array}{l}\text { Atorvastatin } \\
80 \text { mg once daily } \\
\text { and fenofibrate } 54 \\
\text { mg once daily }\end{array}$ & 139 & $\begin{array}{l}\text { Start ezetimibe or } \\
\text { PCSK9 inhibitor }\end{array}$ & $\begin{array}{l}\text { PCSK9 inhibitor not covered } \\
\text { Ezetimibe recommended; } \mathrm{LDL}=72 \mathrm{mg} / \mathrm{dL}\end{array}$ \\
\hline 6 & $\begin{array}{l}\text { General } \\
\text { medicine }\end{array}$ & FH/ASCVD & $\begin{array}{l}\text { Atorvastatin } \\
80 \mathrm{mg} \text { once daily }\end{array}$ & 205 & $\begin{array}{l}\text { Start ezetimibe or } \\
\text { PCSK9 inhibitor }\end{array}$ & $\begin{array}{l}\text { Patient preferred to try oral therapy } \\
\mathrm{LDL}=124 \mathrm{mg} / \mathrm{dL} \text { on ezetimibe } 10 \mathrm{mg} \text { daily }\end{array}$ \\
\hline 7 & Cardiology & $\begin{array}{l}\text { ASCVD; multiple } \\
\text { PCI, statin, } \\
\text { niacin, fenofibrate } \\
\text { intolerance }\end{array}$ & None & 109 & $\begin{array}{l}\text { Start PCSK9 } \\
\text { inhibitor }\end{array}$ & $\begin{array}{l}\text { Approved after physician appeal, but patient } \\
\text { could not afford copay. Patient remains without } \\
\text { lipid-lowering therapy. }\end{array}$ \\
\hline 8 & $\begin{array}{l}\text { General } \\
\text { medicine }\end{array}$ & $\mathrm{FH}$ & $\begin{array}{l}\text { Rosuvastatin } \\
40 \text { mg once daily }\end{array}$ & 180 & $\begin{array}{l}\text { Start ezetimibe or } \\
\text { PCSK9 inhibitor }\end{array}$ & $\begin{array}{l}\text { PCSK9 inhibitor not covered } \\
\text { Ezetimibe recommended; LDL }=97 \mathrm{mg} / \mathrm{dL}\end{array}$ \\
\hline 9 & Endocrinology & $\begin{array}{l}\text { FH/ASCVD/statin } \\
\text { intolerance }\end{array}$ & None & 140 & $\begin{array}{l}\text { Start PCSK9 } \\
\text { inhibitor }\end{array}$ & $\begin{array}{l}\text { Insurance denied; must try ezetimibe } \\
\mathrm{LDL}=200 \mathrm{mg} / \mathrm{dL} \text { on ezetimibe } 10 \mathrm{mg} \text { daily } \\
\text { PCSK9 inhibitor service reconsult; } \\
\mathrm{LDL}=102 \mathrm{mg} / \mathrm{dL} \text { on evolocumab }\end{array}$ \\
\hline
\end{tabular}

a Dosing: alirocumab = $75 \mathrm{mg}$; evolocumab $=140 \mathrm{mg}$ subcutaneous injection every 2 weeks.

ASCVD=atherosclerotic cardiovascular disease; $F H=$ familial hypercholesterolemia; $L D L=$ low-density lipoprotein; $P C I=$ percutaneous coronary intervention.

Table 1 illustrates the experience with the first 9 consultations. Five patients were approved for PCSK9 inhibitor therapy. Patient 1 discontinued alirocumab therapy after approximately 1 month because of itching, chest heaviness, and a pounding headache that occurred 3 hours after taking the medication. The clinical pharmacist assisted the physician with managing these events and determined that the itching and headache may have been a hypersensitivity reaction. The patient was rechallenged after premedication with diphenhydramine and experienced improvement with the itching and headaches but still complained of chest heaviness and pounding. Since chest heaviness is not included as an adverse event in the prescribing guide, this reaction was reported to the FDA MedWatch database. The patient achieved a 35\% LDL reduction, but therapy was subsequently discontinued by the patient.

Patients 2 and 3 were initiated on alirocumab $75 \mathrm{mg}$ subcutaneously every 2 weeks and achieved a reduction in LDL levels of $76 \%$ and $54 \%$, respectively, with no reports of adverse effects. Patient 7 was initially denied coverage by the insurance provider because the patient had not proven intolerance to 2 statins at any dose. The physician submitted an appeal stating that retrial with another statin was inappropriate because of the documentation of severe myositis and intolerance to fibrate and niacin. Approval was received for evolocumab, but the patient was unable to start therapy because of a copay of more than $\$ 300$ per month. Patient 9 was initially denied coverage by the insurance provider because ezetimibe was the preferred agent in the stepped care treatment algorithm provided by the insurance provider. The patient was started on ezetimibe therapy but did not achieve any reduction in LDL level. The service was reconsulted, and the patient was eventually approved for evolocumab therapy and achieved an LDL reduction of $49 \%$.

The remaining patients were either denied, preferred oral therapy, or did not have an approved indication. Patient 4 did not qualify for PCSK9 inhibitor therapy according to the service treatment algorithm, and no additional recommendations were made because of a low LDL level of $86 \mathrm{mg} / \mathrm{dL}$ on a high intensity statin. Patient 5 was indicated for PCSK9 inhibitor therapy but was denied coverage by the insurance provider. The patient was started on ezetimibe adjunct therapy as an alternative recommendation by the consultation service and achieved 48\% LDL reduction. Patient 6 was also indicated for PCSK9 inhibitor therapy but was started on ezetimibe as adjunct therapy because of patient preference and achieved 39\% LDL 
reduction. Patient 8 was indicated for PCSK9 inhibitor therapy but was denied coverage by the insurance provider. Ezetimibe was initiated as an alternative adjunct therapy recommendation by the consultation service, and the patient achieved a $46 \%$ LDL reduction.

\section{Barriers and Limitations}

The biggest challenge in the consultation service development involved the construction of the electronic consult order form, which required content revision before it was approved by the UIH Rules and Orders Committee, and was given several tests before it was ready for full implementation in the EMR system. Limitations to the implementation of the consultation service include (a) inability to capture patients if the pharmaceutical company patient enrollment forms are used rather than initiating a prescription in the EMR and (b) low electronic prescribing rate, possibly because of cost, and the absence of long-term efficacy and safety outcomes.

\section{Implications/Recommendations}

This review highlights the development of a new consultation service model at UIH for the management of the PCSK9 inhibitor agents alirocumab and evolocumab. The model integrates clinical pharmacy services from various outpatient providers with specialty pharmacy services. This partnership may lead to improved access and appropriate management of these medications and the creation of a new revenue stream for the on-site specialty pharmacy.

Health system-based specialty pharmacy practice models have been documented in the literature and have demonstrated benefit to patients and providers. ${ }^{16-20}$ For multiple sclerosis, an integrated model led to improved medication adherence and better communication between the clinic and the specialty pharmacy. ${ }^{17,20}$ In Hanson et al. (2013), the implementation of clinical care guidelines for the safety monitoring of biologic response modifiers resulted in increased compliance with all required laboratory tests. ${ }^{18}$ In this study, the EMR facilitated communication and monitoring of laboratory tests between the clinics and the in-house specialty pharmacy. The pharmacist's role in the treatment of genotype 1 hepatitis $C$ virus was described in Sebhatu and Martin (2016). ${ }^{19}$ In this review, the clinic-based pharmacist performed a comprehensive evaluation of patients in the hepatitis $\mathrm{C}$ clinic before referral to a specialty pharmacy for PA.

The literature describing integrated clinic and specialty pharmacy models offers justification and support for the development of our PCSK9 inhibitor consultation service. This service differs from other integrated models described in the literature in that the PCSK9 inhibitor service incorporated a linked consult pool in the EMR so that any provider in the health system who enters a prescription is prompted to select the consultation service. This feature increases the virtual presence of the consultation service throughout the health system.
The PCSK9 inhibitor consultation service allows clinical pharmacists embedded in outpatient clinics to proactively evaluate patients for therapeutic appropriateness. Specialty pharmacies can focus on PA processing, treatment initiation, training, and medication delivery effectively, while allowing the clinical pharmacists to dedicate their time and resources to direct patient care in the clinics.

This practice model also demonstrates the capabilities of an integrated EMR to implement a consultation system for specialty medications that improves communication and service workflow. Linked prompts in the EMR capture consult orders at the point of prescribing. However, the content length, number of user steps, and the ability to link consult orders are all items that need to be considered. Pharmacy services using shared pools and EMR systems must ensure adequate training of personnel in order to avoid violating HIPAA privacy rules.

The prescribing of PCSK9 inhibitors has been lower than anticipated at UIH. The uptake of these agents by providers may be hampered by the lack of long-term efficacy and safety data and cost of the agents. In addition, there is concern regarding the increase in neurocognitive events, such as, amnesia, memory impairment, and confusional state, all of which has been noted in alirocumab and evolocumab landmark trials. ${ }^{3,4}$

However, the neurocognitive effects associated with PCSK9 inhibitors have not been associated with achieving low LDL levels, since patients who achieved LDL levels of less than 25 $\mathrm{mg} / \mathrm{dL}$ in the Efficacy and Safety of Alirocumab in Reducing Lipids and Cardiovascular Events (ODYSSEY LONG TERM) trial did not experience an increase in neurocognitive effects when compared with placebo. ${ }^{3}$ The same trend was noted in the Efficacy and Safety of Evolocumab in Reducing Lipids and Cardiovascular Events (OSLER) trial. ${ }^{4}$ The investigators of the ODYSSEY LONG TERM trial highlighted the absence of a formal neurocognitive test as a limitation of their study design, and the OSLER trial had an open label study design, which may have introduced possible bias with the reporting of adverse effects. ${ }^{3,4}$

The results of the Evaluation of Cardiovascular Outcomes After an Acute Coronary Syndrome During Treatment with Alirocumab (ODYSSEY OUTCOME) trial, which is scheduled to be completed in 2018, will provide long-term data on the cardiovascular outcomes relating to alirocumab therapy. ${ }^{21}$ The results of the Evaluating Effect of the Study Drug Alirocumab on Neurocognitive Function When Compared to Placebo trial is scheduled to be completed in 2020 and will provide longterm safety data regarding the potential effect of alirocumab on neurocognitive health. ${ }^{22}$

Results from the Further Cardiovascular Outcomes Research with PCSK9 Inhibition in Subjects with Elevated Risk (FOURIER) trial and the Evaluating PCSK9 Binding Antibody Influence on Cognitive Health in High Cardiovascular Risk Subjects (EBBINGHAUS) trial, which was a double blind 
placebo study, were released in March 2017. ${ }^{23,24}$ Evolocumab met its primary composite outcome of time to cardiovascular death, myocardial infarction, hospitalization from unstable angina, stroke or coronary revascularization in the FOURIER trial and its primary outcome of mean change from baseline over time in the spatial working memory index of executive function in the EBBINGHAUS trial. ${ }^{24,25}$ The results of these studies, as well as the indication for the treatment of homozygous $\mathrm{FH}$, could potentially increase the prescribing of evolocumab in the PCSK9 inhibitor market.

The consultation service project presented here showcases the benefits of collaboration between clinic-based pharmacists and specialty pharmacies and highlights areas for possible pharmacy residency training opportunities. The PCSK9 inhibitor consultation service will continue to provide service to the UIH health system and capture valuable data regarding this novel class of specialty medications.

The UIH PCSK9 inhibitor consultation service was successfully implemented with a clinical review process, EMR capabilities, and collaboration with the on-site specialty pharmacy to provide a unique and comprehensive proactive patient evaluation and management service. The service facilitates medication management from the point of prescribing, patient access, administration, and monitoring for this class of specialty medications. This service model could be replicated in other health systems for the management of patients eligible for PCSK9 inhibitor therapy. Lessons learned could be disseminated for the development of similar models for other specialty medication management opportunities.

\section{Authors}

ADENIKE ATANDA, PharmD, CTTS, BCACP, University of North Texas System College of Pharmacy, Fort Worth, Texas. NANCY L. SHAPIRO, PharmD, FCCP, BCACP; JOANN STUBBINGS, BSPharm, MHCA; and VICKI GROO, PharmD, University of Illinois at Chicago College of Pharmacy, Chicago, Illinois.

AUTHOR CORRESPONDENCE: Vicki Groo, PharmD, Clinical Associate Professor, Dept. of Pharmacy, University of Illinois at Chicago College of Pharmacy, 833 S. Wood St., Rm. 164, Chicago, IL 60612. Tel.: 312.996.7886; E-mail: vjust@uic.edu.

\section{DISCLOSURES}

No outside funding supported this study. Groo reports speaker bureau fees from Pfizer and Bristol-Myers Squibb. The other authors have nothing to disclose.

All the authors contributed to study concept and design. Atande took the lead in data collection, and data interpretation was performed by Groo and Atanda. The manuscript was written by Atanda and revised by all the authors.

\section{ACKNOWLEDGMENTS}

The authors recognize the following people for their contribution to the creation of this service: Rebekah Hanson, PharmD, BCBS; Christine Rash PharmD; Lisa Kumor, PharmD; John Garafalo, PharmD; Jamie Paek, PharmD; Michael Jhattu; Daphne Smith-Marsh, PharmD, CDE; and Paul Stranges, PharmD

\section{REFERENCES}

1. PRALUENT (alirocumab) injection, for subcutaneous use. Sanofi/Regeneron. Revised April 2017. Available at: http://products.sanofi.us/praluent/praluent. pdf. Accessed July 18, 2017.

2. REPATHA (evolocumab) injection, for subcutaneous use. Amgen. Revised July 2016. Available at: http://pi.amgen.com/ /media/amgen/repositorysites/ pi-amgen-com/repatha/repatha_pi_hcp_english.ashx. Accessed July 18, 2017.

3. Robinson JG, Farnier M, Krempf M, et al. Efficacy and safety of alirocumab in reducing lipids and cardiovascular events. N Engl J Med. 2015;372(16):1489-99.

4. Sabatine MS, Giugliano RP, Wiviott SD, et al. Efficacy and safety of evolocumab in reducing lipids and cardiovascular events. N Engl J Med. 2015;372(16):1500-09.

5. Roth EM, Taskinene MR, Ginsberg HN, et al. Monotherapy with the PCSK9 inhibitor alirocumab versus ezetimibe in patients with hypercholesterolemia: results of a 24 week, double-blind, randomized phase 3 trial. Int J Cardiol. 2014;176(1):55-61.

6. Koren MJ, Lundgvist P, Bolognese M, et al. Anti-PCSK9 monotherapy for hypercholesterolemia: the MENDEL-2 randomized, controlled phase III clinical trial of evolocumab. J Am Coll Cardiol. 2014;63(23):2531-40.

7. Blom DJ, Hala T, Bolognese M, et al. A 52-week placebo-controlled trial of evolocumab in hyperlipidemia. N Engl J Med. 2014;370(19):1809-19.

8. Moriarty PM, Thomason PD, Cannon CP. Efficacy and safety of alirocumab vs ezetimibe in statin-intolerant patients, with a statin rechallenge arm: the ODYSSEY ALTERNATIVE randomized trial. J Clin Lipidol. 2015;9(6):758-69.

9. Stone NJ, Robinson J, Lichtenstein AH, et al. 2013 ACC/AHA guideline on the treatment of blood cholesterol to reduce atherosclerotic cardiovascular risk in adults: a report of the American College of Cardiology/ American Heart Association Task Force on Practice Guidelines. Circulation. 2014;129(25 Suppl 2):S1-45.

10. Taylor F, Huffman MD, Macedo AF, et al. Statins for the primary prevention of cardiovascular disease. Cochrane Database Syst Rev. 2013;1:CD004816.

11. Cholesterol Treatment Trialists Collaboration, Mihaylova B, Emberson J, et al. The effects of lowering LDL cholesterol with statin therapy in people at low risk of vascular disease: meta-analysis of individual data from 27 randomised trials. Lancet. 2012;380(9841):581-90.

12. ACCORD Study Group, Ginsberg HN, Elam MB, et al. Effects of combination lipid therapy in type 2 diabetes mellitus. N Engl J Med. 2010;362(17):1563-74.

13. Cannon CP, Blazing MA, Giugliano RP. Ezetimibe added to statin therapy after acute coronary syndromes. N Engl J Med. 2015;372(25):2387-97.

14. Lloyd-Jones DM, Morris PB, Ballantyne CM, et al. 2016 ACC expert consensus decision pathway on the role of non-statin therapies for LDLcholesterol lowering in the management of atherosclerotic cardiovascular disease risk: a report of the American College of Cardiology Task Force on Clinical Expert Consensus Documents. J Am Coll Cardiol. 2016; 68(1):92-125.

15. Aggarwal S, Loomba RS, Arora RR. Efficacy of colesevelam on lowering glycemia and lipids. J Cardiovasc Pharmacol. 2012;59(2):198-205.

16. Bhat $S$, Khamo N, Abdou S, et al. The pharmacist's role in biologic management for IBD in a health system-integrated practice model. Am J Pharm Benefits. 2015;7(5):215-20. 
17. Hanson RL, Habibi M, Khamo N, et al. Integrated clinical and specialty pharmacy practice model for management of patients with multiple sclerosis. Am J Health Syst Pharm. 2014;71(6):463-69.

18. Hanson RL, Gannon MJ, Khamo N, et al. Improvement in safety monitoring of biologic response modifiers after the implementation of clinical care guidelines by a specialty pharmacy service in an academic health system. J Manag Care Pharm. 2013;19(1):49-67. Available at: http://www.jmcp. org/doi/10.18553/jmcp.2013.19.1.49.

19. Sebhatu P, Martin MT. Genotype 1 hepatitis $C$ virus and the pharmacist's role in treatment. Am J Health Syst Pharm. 2016;73(11):764-74.

20. Habibi M, Kuttab HM. Management of multiple sclerosis and the integration of related specialty pharmacy programs within health systems. Am J Health Syst Pharm. 2016;73(11):811-19.

21. OSYSSEY Outcomes: Evaluation of Cardiovascular Outcomes after an Acute Coronary Syndrome During Treatment with Alirocumab. Clinicaltrials.gov Identifier NCT01663402. Available at: https://clinicaltrials.gov/ct2/show/NCT01663402?term=odyssey+outcome\&rank=1. Accessed July 18, 2017.
22. Evaluation Effect of the Study Drug Praluent (Alirocumab) on Neurocognitive Function When Compared to Placebo. Clinicaltrials.gov Identifier NCT02957682. Available at: https://clinicaltrials.gov/ct2/show/ NCT02957682. Accessed July 18, 2017.

23. Further Cardiovascular Outcomes Research with PCSK9 Inhibition in Subjects with Elevated Risk (FOURIER). ClinicalTrials.gov Identifier NCT01764633. Available at: https://clinicaltrials.gov/ct2/show/NCT0176463 3?term=fourier+study\&rank=2. Accessed July 18, 2017.

24. Evaluating PCSK9 Binding antiBody Influence oN coGnitive HeAlth in High cardiovascUlar Risk Subjects (EBBINGHAUS). ClinicalTrials.gov Identifier NCT02207634. Available at: https://clinicaltrials.gov/ct2/show/ NCT02207634?term=ebbinghaus\&rank=1. Accessed July 18, 2017.

25. Amgen. Amgen announces Repatha (evolocumab) significantly reduced the risk of cardiovascular events in FOURIER outcomes study. Cision PR Newswire. February 2, 2017. Available at: http://www.prnewswire.com/ news-releases/amgen-announces-repatha-evolocumab-significantly-reducedthe-risk-of-cardiovascular-events-in-fourier-outcomes-study-300401508. html. Accessed July 18, 2017. 